\title{
Mejoramiento del desempeño de paneles fotovoltaicos a partir de la irrigación forzada de la superficie superior
}

\section{Improvement of the performance of photovoltaics panels by forced irrigation on the upper surface}

\author{
German Osma $^{1}$, Carlos Florez ${ }^{2}$, Wilmer Rojas ${ }^{3}$, Julián Florez ${ }^{4}$, Gabriel Ordoñez ${ }^{5}$ \\ ${ }^{1}$ Grupo de Investigación en Sistemas de Energía Eléctrica - GISEL, Escuela de Ingeniería \\ Eléctrica, Electrónica y de Telecomunicaciones, Universidad Industrial de Santander, Colombia. \\ Email: gealosma@uis.edu.co \\ ${ }^{2}$ Electro Sotfware, Bucaramanga, Colombia. Email: carlos.florez2@ correo.uis.edu.co \\ ${ }^{3}$ Acsendo, Bogotá, Colombia. Email: wilmer.rojas@ correo.uis.edu.co \\ ${ }^{4}$ Grupo de Investigación en Sistemas de Energía Eléctrica - GISEL, Escuela de Ingeniería \\ Eléctrica, Electrónica y de Telecomunicaciones, Universidad Industrial de Santander, Colombia. \\ Email: florezjulian87@gmail.com \\ ${ }^{5}$ Grupo de Investigación en Sistemas de Energía Eléctrica - GISEL, Escuela de Ingeniería \\ Eléctrica, Electrónica y de Telecomunicaciones, Colombia. Email: gaby@uis.edu.co
}

RECIBIDO: MAYO 29, 2016. ACEPTADO: MARZO 11, 2017. VERSIÓN FINAL: MAYO 17, 2017.

\section{RESUMEN}

La irrigación de la superficie superior de los paneles fotovoltaicos $(\mathrm{FV})$ es uno de los métodos más efectivos para reducir su temperatura de operación e incrementar la potencia generada. Aunque existen publicaciones sobre esta técnica, hay poca información sobre el efecto de las variaciones del tiempo de irrigación y caudal de riego. Este trabajo presenta un estudio experimental sobre el efecto de estos dos factores para un panel FV de $255 \mathrm{~W}$ instalado en un entorno de clima cálido tropical. Se encontró que caudales de $4.5 \mathrm{l} / \mathrm{min}$ o superiores pueden producir incrementos de hasta $10 \%$ en la energía generada adicional diaria; adicionalmente, que los tiempos de irrigación tienen una significativa influencia en la disminución de la temperatura de operación promedio y la energía adicional generada por un panel FV.

Palabras Clave: Generación fotovoltaica, Irrigación, Mitigación térmica.

\begin{abstract}
Irrigation on upper surface of the PV panels is one of the most effective methods to reduce the operating temperature and to increase the PV power. Although there are publications about this technique, there is little information about the effect of the time and irrigation flow variations on the PV performance. This article presents an experimental study about the effect of both factor for a PV panel of $255 \mathrm{~W}$ installed in an warm - tropical place. It was found that flows of $4.5 \mathrm{l} / \mathrm{min}$ o higher can produce increases up to $10 \%$ in additional generated energy per day; in addition, irrigation time has a significant influence on the average operating temperature decreases and additional generated energy per day.
\end{abstract}

KEYWORDS: PV system, Irrigation, Thermal mitigation.

Este artículo puede compartirse bajo la licencia CC BY-ND 4.0 y se referencia usando el siguiente formato: G. Osma, C. Flórez, W. Zambrano, J. Flórez, G. Ordóñez, "Mejoramiento del desempeño de paneles fotovoltaicos a partir de la irrigación forzada de la superficie superior", UIS Ingenierías, vol. 16, no. 2, pp. 161-172, Julio-Diciembre 2017. Doi: https://doi.org/10.18273/revuin.v16n2-2017015 


\section{INTRODUCCIÓN}

La energía solar fotovoltaica es una de las aplicaciones energéticas verdes más utilizadas en las edificaciones urbanas [1].

El rendimiento de un sistema fotovoltaico (FV) depende tanto de sus características técnicas (tensión de máxima potencia, corriente de máxima potencia, potencia máxima, tensión de circuito abierto y corriente de corto circuito, entre otros) como de la irradiación solar incidente y la temperatura de operación, entre otros [2].

Específicamente, el aumento de la temperatura ocasiona la reducción de la tensión de circuito abierto y la tensión de máxima potencia, y en consecuencia disminuye la potencia máxima generada por el panel FV [3], [4]. Esto se presenta en mayor grado para niveles altos de irradiación solar $\left(>600 \mathrm{~W} / \mathrm{m}^{2}\right)$ y de temperatura del aire circundante $\left(>25^{\circ} \mathrm{C}\right)$, la cual depende de la temperatura ambiente y del emplazamiento de instalación del sistema FV.

$\mathrm{Ju}$ y $\mathrm{Fu}$ [5] determinaron que un aumento de $1{ }^{\circ} \mathrm{C}$ en la temperatura de operación causa la disminución de la eficiencia de generación de energía en aproximadamente en $0,5 \%$.

Se estima que la eficiencia energética de los paneles FV puede ser reducida hasta en un $15 \%$ debido al aumento de la temperatura del panel FV en un entorno tropical cálido [6].

Estas pérdidas de potencia generada pueden ser mitigadas a partir de la utilización de algunas estrategias de climatización, tales como techo verde debajo del panel FV [7], [8], ventilación forzada [9], [10] e irrigación forzada de la superficie superior del panel FV [5], [11][15], entre otras.

La circulación de aire natural o forzado es un método simple y de bajo costo para disminuir el calor de los paneles FV, sobre todo para lugares cuya temperatura ambiente es superior a $20{ }^{\circ} \mathrm{C}$ [2]. Sin embargo, es una estrategia de impacto intermitente debido a la variabilidad del aire y que favorece en general sólo a los paneles FV más externos en dirección al viento.

Por otro lado, Lamnatou y Chemisana [7], [8] estudiaron el efecto benéfico de los techos verdes como factor mitigador de la temperatura de operación de un panel FV. Específicamente, concluyeron que un techo verde puede aumentar la eficiencia de conversión del panel FV entre $1,3 \%$ y $3,3 \%$.

La extracción de calor de un panel FV por medio del agua es más costosa que la extracción por aire, aunque puede ser más efectiva [2]. En países como Arabia Saudita, Irak, Egipto, Australia y China, entre otros, se han desarrollado estudios usando el agua como refrigerante del panel FV, considerando la irrigación por goteo [13], chorros [16] o película de agua [11].

La irrigación de un panel FV permite disipar el calor debido a la pérdida de calor por convección entre el agua y la superficie superior del panel FV, lo cual produce un aumento en la potencia eléctrica de salida [2].

Krauter [6] encontró que para un sitio con condiciones similares a Rio de Janeiro se puede lograr una ganancia de eficiencia energética diaria del 8,9\% por el bombeo de agua sobre la superficie del panel. La irrigación empleada en este experimento fue tipo película en la superficie frontal.

Reindl et al. [17] concluyeron que la mitigación de la temperatura de operación del FV debe ser una prioridad en zonas tropicales cálidas (cerca al Ecuador) debido a la alta radiación solar incidente sostenida durante el año.

Habiballahi [18] desarrolló un experimento considerando tres caudales de irrigación en litros por minuto $(2,97$ 1/min, 4,57 1/min y 5,17 1/min). Encontró que en condiciones de alta radiación solar el incremento de la potencia generada puede ser de hasta $20 \%$ en un instante dado, y que tal incremento es menor a medida que el nivel de radiación solar disminuye, por lo que en promedio se puede indicar que el incremento diario será cercano al $10 \%$.

La Tabla 1 presenta investigaciones realizadas utilizando el agua como refrigerante en paneles FV. La mayoría de estudios se llevaron a cabo en entornos no tropicales. Los incrementos en la generación de energía oscilaron mayormente entre $4 \%$ y $10 \%$, para los cuales se considera mayormente la irrigación por flujo constante con caudales entre $2 \mathrm{l} / \mathrm{min}$ y $7 \mathrm{l} / \mathrm{min}$.

Teniendo en cuenta lo mencionado, y que la ciudad de Bucaramanga tiene un clima tropical-cálido con temperatura ambiente de hasta $32^{\circ} \mathrm{C}$ y radiación solar diaria promedio de $4,9 \mathrm{kWh} / \mathrm{m}^{2}$ [20], es de interés estudiar el potencial beneficio de la irrigación en la generación FV. Se estima que la reducción de la potencia generada en esta ciudad puede ser de hasta $15 \%$ debido al calentamiento del panel FV.

Por tanto, el objeto de este trabajo es analizar el impacto de la técnica de irrigación como estrategia de mitigación térmica en un panel FV instalado sobre un techo verde en operación en un entorno tropical cálido. Principalmente, se estudia el efecto de diversos tiempos de irrigación, dado que en la literatura se abordan regímenes de irrigación continuos. 
El trabajo realizado expone inicialmente la descripción de la experimentación realizada (Sección II), posteriormente, muestra el análisis de resultados (Sección III) y finalmente presenta las conclusiones (Sección IV).

Tabla 1. Estudios realizados sobre el impacto de la irrigación de agua en paneles FV.

\begin{tabular}{ccccccc}
\hline & & & \multicolumn{3}{c}{ Temperatura $\left({ }^{\circ} \mathrm{C}\right)$} & Aumento \\
\cline { 5 - 7 } Ref. & País & $\begin{array}{c}\text { Lat. } \\
\left({ }^{\circ}\right)\end{array}$ & Amb. & $\begin{array}{c}\text { Sin } \\
\text { riego }\end{array}$ & $\begin{array}{c}\text { Con } \\
\text { riego }\end{array}$ & $\begin{array}{c}\text { efiencia } \\
{[\%]}\end{array}$ \\
\hline$[6]$ & Brasil & $-22,9$ & 20,0 & 60 & 38 & 10,3 \\
\hline$[15]$ & Irán & 30,3 & $25-34$ & $25-59$ & $25-36$ & 3,66 \\
\hline$[13]$ & Australia & $-32,0$ & $22-25$ & 58 & 40 & $4-10$ \\
\hline$[18]$ & Irán & 30,0 & $30-34$ & $34-61$ & $33-41$ & 20,0 \\
\hline$[12]$ & Arabia & 26,3 & 20 & 37,8 & 30,5 & 9,0 \\
\hline$[14]$ & Sudáfrica & $-30,6$ & 26 & $45-59$ & $30-34$ & 3,6 \\
\hline$[19]$ & USA & 45,5 & $17-25$ & 55 & 40 & 8,3 \\
\hline$[16]$ & Arabia & 26,3 & $25-35$ & $30-79$ & 30 & 9,0 \\
\hline$[2]$ & Irak & 33,2 & $30-35$ & 76,8 & 70,1 & 9,8 \\
\hline
\end{tabular}

Fuente. Elaboración propia.

\section{DESCRIPCIÓN DE LA EXPERIMENTACIÓN}

El experimento se llevó a cabo en el Edificio de Ingeniería Eléctrica de la Universidad Industrial de Santander, ubicada en la ciudad de Bucaramanga $\left(7,13^{\circ}\right.$ latitud norte y $73,13^{\circ}$ longitud oeste). Esta edificación es un piloto de edificación verde utilizado para el desarrollo de investigación en generación renovable, uso racional de la energía y automatización, entre otros.

Este edificio cuenta con una capacidad instalada de generación fotovoltaica de $7,5 \mathrm{~kW}$ a partir de dos sistemas FV (FV1 y FV2). Para este experimento se utilizaron paneles FV del sistema FV2 mostrado en la Figura 1. Este sistema de inyección a la red eléctrica está conformado por 10 paneles FV de 255W Canadian Solar que son gestionados por microinversores Enphase M250.

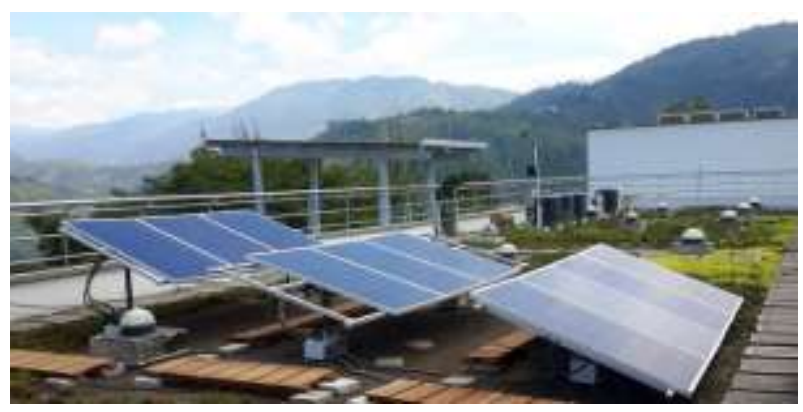

Figura 1. Sistema FV2 de inyección a la red. Fuente. Elaboración propia.

Específicamente, se utilizaron los paneles FV P5, P6 y P7 del sistema FV2. El panel P5 no fue irrigado y se tomó como la referencia de temperatura de operación y potencia generada. Con respecto a estas variables de desempeño, se determinó el efecto de la irrigación en los paneles $\mathrm{P} 6$ y P7, los cuales fueron irrigados para diversos regímenes de operación.

La Figura 2 muestra el sistema de riego de los paneles $\mathrm{FV}$, que consta de 1 bomba de recirculación de agua para llevar el agua del tanque de acopio a la parte superior del panel FV, 4 sensores de temperatura instalados en la parte posterior de los paneles $\mathrm{FV}, 1$ electroválvula para el llenado del tanque o la irrigación directa del panel FV, 1 tanque de acopio, 1 controlador para gestionar la operación de la bomba y la electroválvula.

El sistema operó con bombas de $8 \mathrm{~W}$ para suministrar caudales que variaron entre $1.8 \mathrm{l} / \mathrm{min}$ y $9.0 \mathrm{l} / \mathrm{min}$.

La monitorización de variables no eléctricas se llevó a cabo por medio de un conjunto de sensores. La radiación solar se monitorizó por medio un piranómetro Kipp\&Zonen SMP11 (patrón secundario) conectado a un datalogger CAMPBELL CR800X; mientras la temperatura del panel $\mathrm{FV}$ se obtuvo por medio de termocuplas OMEGA 5TC-TT-KI-36-2M y una datalogger OMEGA OM-CP-OCTTEMP2000.

La medición del nivel de agua en el tanque de acopio fue realizada por un sensor de distancia de ultrasonido HCSR04 capaz de detectar objetos y calcular la distancia a la que se encuentra en un rango de $2 \mathrm{~cm}$ a $450 \mathrm{~cm}$.

La medición de las variables eléctricas, tensión, corriente, potencia y energía, se llevó a cabo por medio de medidores de energía DC y AC, AcuDC243 y AcuRev 2020, respectivamente; ambos de clase 0,5. La Figura 3 muestra el esquema de conexión de los medidores. La Figura 4 presenta el tablero de protecciones eléctricas y medidores DC de los paneles FV P5, P6 y P7. 


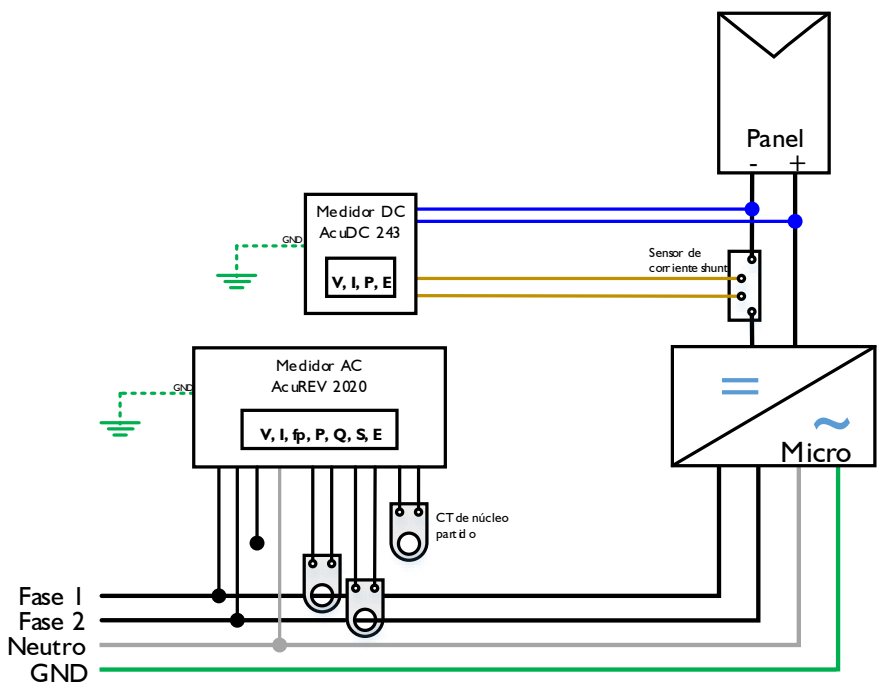

Figura 3. Esquema de conexión de medidores DC y AC. Fuente. Elaboración propia.

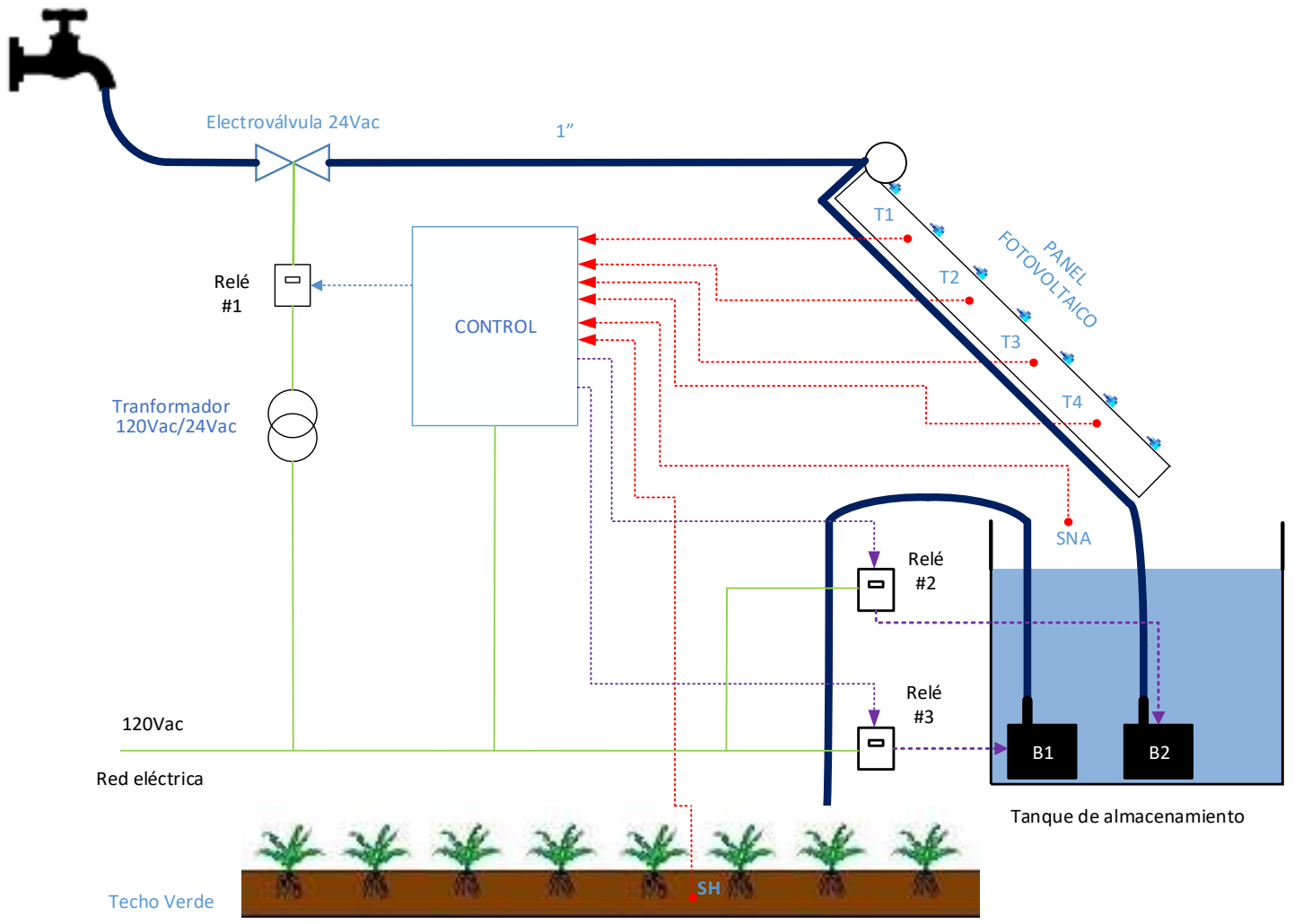

Figura 2. Esquema del sistema de irrigación de los paneles FV. Fuente. Elaboración propia. 


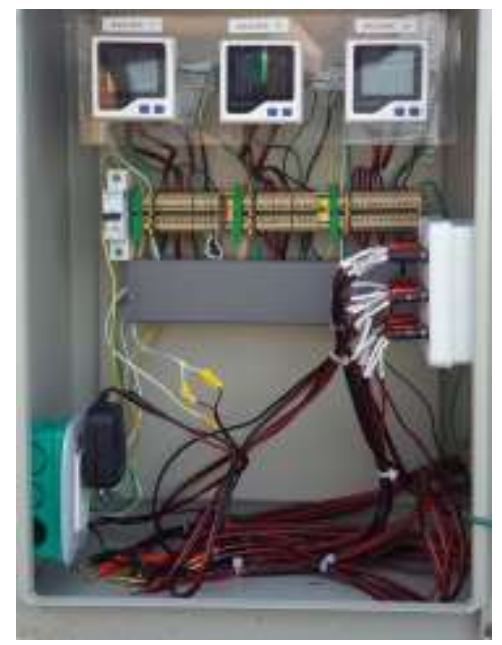

Figura 4. Vista interior del tablero DC. Fuente. Elaboración propia.

La experimentación se realizó durante un lapso de 1 mes para los siete casos analizados, que la Tabla 2 presenta. El tiempo total de operación se estableció en 30 minutos, que cobija el tiempo de irrigación y el tiempo de no irrigación; por ejemplo, en el Caso 1 el panel P6 tiene un régimen de 15 minutos de irrigación y 15 minutos de no irrigación. Se aclara que el Caso VII consistió en una irrigación permanente de los paneles FV P6 y P7.

Tabla 2. Casos de irrgación ejecutados.

\begin{tabular}{|c|c|c|c|c|c|}
\hline \multicolumn{3}{|c|}{ Panel FV P6 } & \multicolumn{3}{|c|}{ Panel FV P7 } \\
\hline Riego & $\begin{array}{c}\text { Tiempo } \\
\text { [min] }\end{array}$ & $\begin{array}{l}\text { Caudal } \\
{[1 / \mathrm{min}]}\end{array}$ & Riego & $\begin{array}{c}\text { Tiempo } \\
\text { [min] }\end{array}$ & $\begin{array}{l}\text { Caudal } \\
{[1 / \mathrm{min}]}\end{array}$ \\
\hline 1 Película & $15^{\prime}: 15^{\prime}$ & 9,5 & Película & $1^{\prime}: 29^{\prime}$ & 9,5 \\
\hline 2 Película & $15^{\prime}: 15^{\prime}$ & 9,5 & $\begin{array}{c}\text { Chorros } \\
10 \mathrm{~cm}\end{array}$ & $15^{\prime}: 15^{\prime}$ & 3,8 \\
\hline $\begin{array}{c}\text { Chorros } \\
10 \mathrm{~cm}\end{array}$ & $15^{\prime}: 15^{\prime}$ & 3,8 & $\begin{array}{c}\text { Chorros } \\
20 \mathrm{~cm}\end{array}$ & $15^{\prime}: 15^{\prime}$ & 1,8 \\
\hline $\begin{array}{c}\text { Chorros } \\
10 \mathrm{~cm}\end{array}$ & $1^{\prime}: 29^{\prime}$ & 3,8 & $\begin{array}{c}\text { Chorros } \\
20 \mathrm{~cm}\end{array}$ & $1^{\prime}: 29^{\prime}$ & 1,8 \\
\hline 5 Película & $15^{\prime}: 15^{\prime}$ & 4,5 & Película & $1^{\prime}: 29 '$ & 4,5 \\
\hline 6 Película & $1^{\prime}: 29$ ' & 9,5 & $\begin{array}{c}\text { Chorros } \\
10 \mathrm{~cm} \\
\end{array}$ & $1^{\prime}: 29^{\prime}$ & 3,8 \\
\hline 7 Película & Perm. & 9,5 & Película & Perm. & 4,5 \\
\hline
\end{tabular}

Fuente. Elaboración propia.

La Figura 5 presenta el estado de los paneles FV P6 y P7 cuando son irrigados. El panel FV P5 al no ser irrigado es la referencia que permite cuantificar el efecto de la irrigación en la temperatura de operación y la potencia generada.

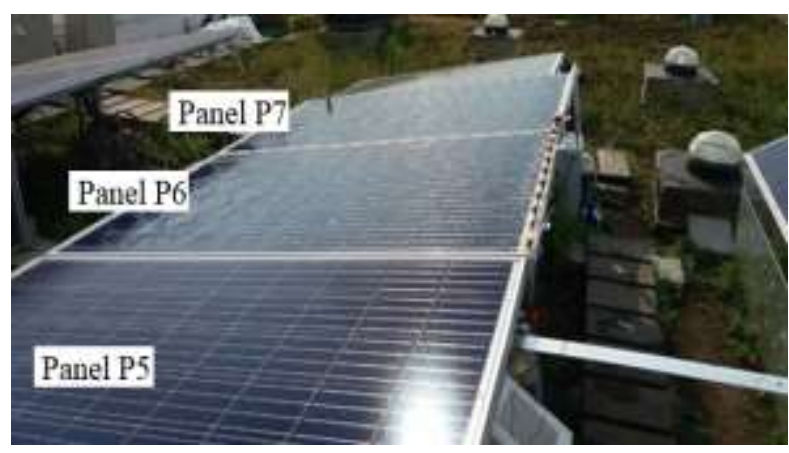

Figura 5. Paneles FV en estado de irrigación. Fuente. Elaboración propia.

\section{ANÁLISIS DE RESULTADOS}

El impacto de la irrigación sobre la superficie superior del panel FV se describe a partir de la variación de la curvas de temperatura de operación y la potencia generada con respecto al comportamiento del panel FV P5 (referencia). Tal análisis gráfico se realiza en este artículo para los casos 1 y 7.

La Figura 6 muestra el comportamiento de las temperaturas de operación de los paneles FV P5, P6 y P7, a partir de las curvas $T p 5, T p 6$ y $T p 7$, respectivamente, entre 9 a.m. y 3 p.m.

Se aprecia como la curva $T p 5$ es el escenario más desfavorable y es influenciada por la radiación solar (Rad. Solar), siendo ésta el límite superior para las curvas Tp6 y Tp7. Por otro lado, la temperatura ambiente (Tamb) es el límite inferior y condición deseada.

Las curvas Tp6 y $T p 7$ muestran que el primer minuto de irrigación produce la mayor parte del decremento de la temperatura de operación de los paneles FV P6 y P7. Asimismo, se aprecia que la temperatura de los paneles FV aumenta nuevamente posterior a la irrigación.

El decremento de temperatura que produce la irrigación es mayor cuando el nivel de irradiación solar incidente es alto $\left(>600 \mathrm{~W} / \mathrm{m}^{2}\right)$, debido a que el panel FV experimenta una mayor temperatura de operación.

Se aprecia la irrigación no logra que la temperatura de operación de los paneles FV P6 y P7 descienda a la temperatura ambiente. Esto se debe a que la temperatura del agua es mayor en algunos grados $\left(2^{\circ} \mathrm{C}\right.$ a $\left.5^{\circ} \mathrm{C}\right)$ a la temperatura ambiente, lo cual se debe al proceso de transferencia de calor entre el panel FV y el agua de irrigación.

La Figura 7 muestra las curvas de decremento de la temperatura para los paneles FV P6 y P7, $4 T p 5 p 6$ y 
$\triangle T p 5 p 7$, con respecto a la temperatura $T p 5$. Se aprecia que la irrigación puede reducir hasta en $20^{\circ} \mathrm{C}$ la temperatura de un panel $\mathrm{FV}$ para altos niveles de radiación solar. El decremento es no mayor a $10^{\circ} \mathrm{C}$ para una radiación solar sostenida menor de $500 \mathrm{~W} / \mathrm{m}^{2}$, y es menor a $2^{\circ} \mathrm{C}$ para una irradiación menor a $200 \mathrm{~W} / \mathrm{m}^{2}$.

La Figura 8 describe el impacto de la irrigación en la potencia generada, específicamente las curvas $\triangle P p 6 p 5$ y $\triangle P p 7 p 5$ muestran la potencia adicional obtenida con respecto a la potencia generada por el panel de referencia.

El máximo incremento de potencia en cada ciclo de operación puede ser de hasta $30 \mathrm{~W}$ para altas radiaciones solares y no superiror a $10 \mathrm{~W}$ cuando la radiación es menor a $500 \mathrm{~W} / \mathrm{m}^{2}$.

Las curvas $\triangle P p 6 p 5$ y $\triangle P p 7 p 5$ permiten apreciar el incremento inmediato de la potencia generada en relación a la variación de las curvas $\Delta T p 5 p 6$ y $\Delta T p 5 p 7$.

De manera similar a las figuras 6,7 y 8 , se describe el Caso 7 a partir de las figuras 9,10 y 11 para los dos regímenes permanentes con caudales de 4,5 1/min y 9,5 $1 / \mathrm{min}$.
Las curvas $T p 6, T p 7, \Delta T p 5 p 6$ y $\Delta T p 5 p 7$ muestran que los dos caudales producen un efecto similar. Esto permite ajustar el dimensionamiento del sistema de irrigación para una bomba que suministre a lo sumo un caudal 4,5 1/min, siendo de menor potencia eléctrica y ocasionando un menor consumo energético.

A pesar de mantener la irrigación de forma permanente, no se logra disminuir la temperatura de operación de los paneles FV al nivel de la temperatura ambiente. Esto sucede por el calentamiento del fluido debido al proceso de transferencia de calor del panel FV y la radiación solar sobre la capa de agua de irrigación.

Sin embargo, se aprecia que se puede obtener una reducción de la temperatura de operación superior a $20^{\circ} \mathrm{C}$ para una condición de alta irradiación solar incidente y sostenida. Tal disminución oscila entre $0^{\circ} \mathrm{C}$ y $3^{\circ} \mathrm{C}$ cuando la radiación solar es menor a $200 \mathrm{~W} / \mathrm{m}^{2}$.

Con respecto al impacto en la potencia generada, se observa que es posible obtener una beneficio superior a los $30 \mathrm{~W}$ para altos niveles de irradiación solar, y que en promedio el incremento de potencia es cercano a $10 \mathrm{~W}$ lo que permite que la energía adicional generada sea de hasta $10 \%$.

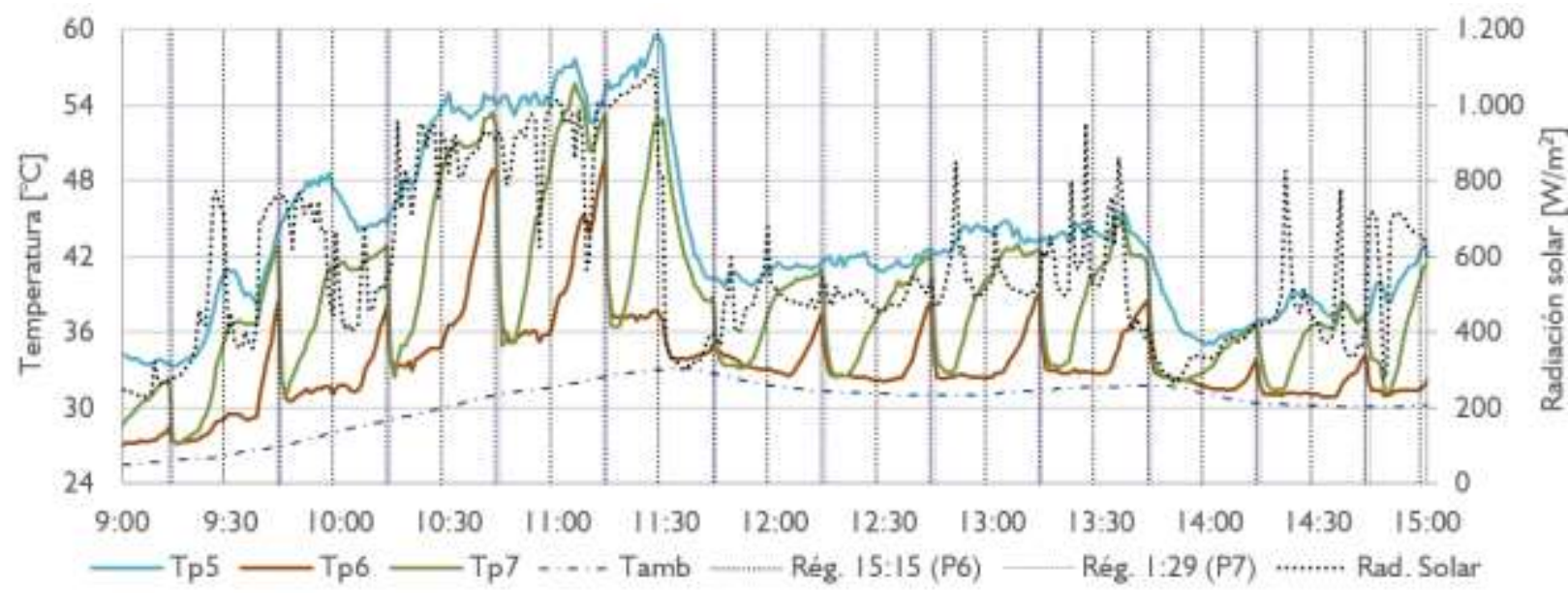

Figura 6. Curvas de las temperaturas de operación de las paneles FV del Caso I. Fuente. Elaboración propia. 


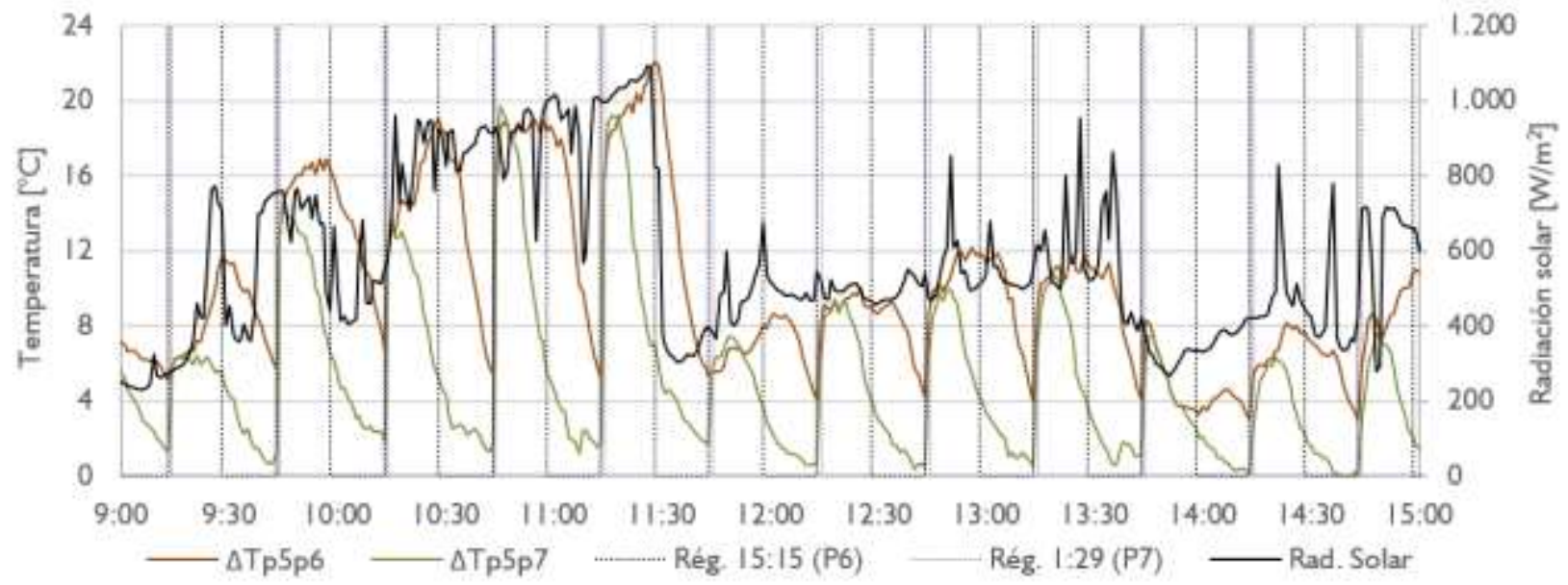

Figura 7. Curvas de decremento de la temperatura de operación de los paneles FV P6 y P7 del Caso I con respecto a la temperatura de operación del panel FV P5. Fuente. Elaboración propia.

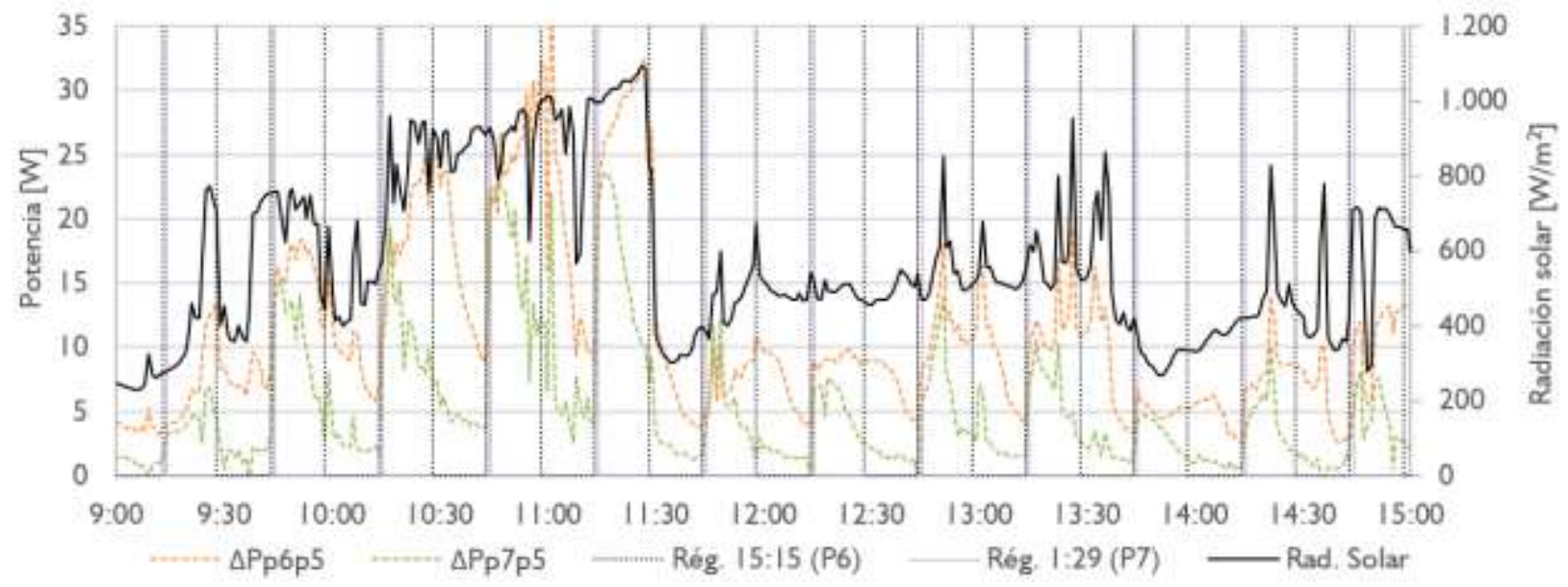

Figura 8. Curvas de incremento de la potencia generada de los paneles FV P6 y P7 del Caso I con respecto a la potencia generada por el panel FV P5. Fuente. Elaboración propia. 


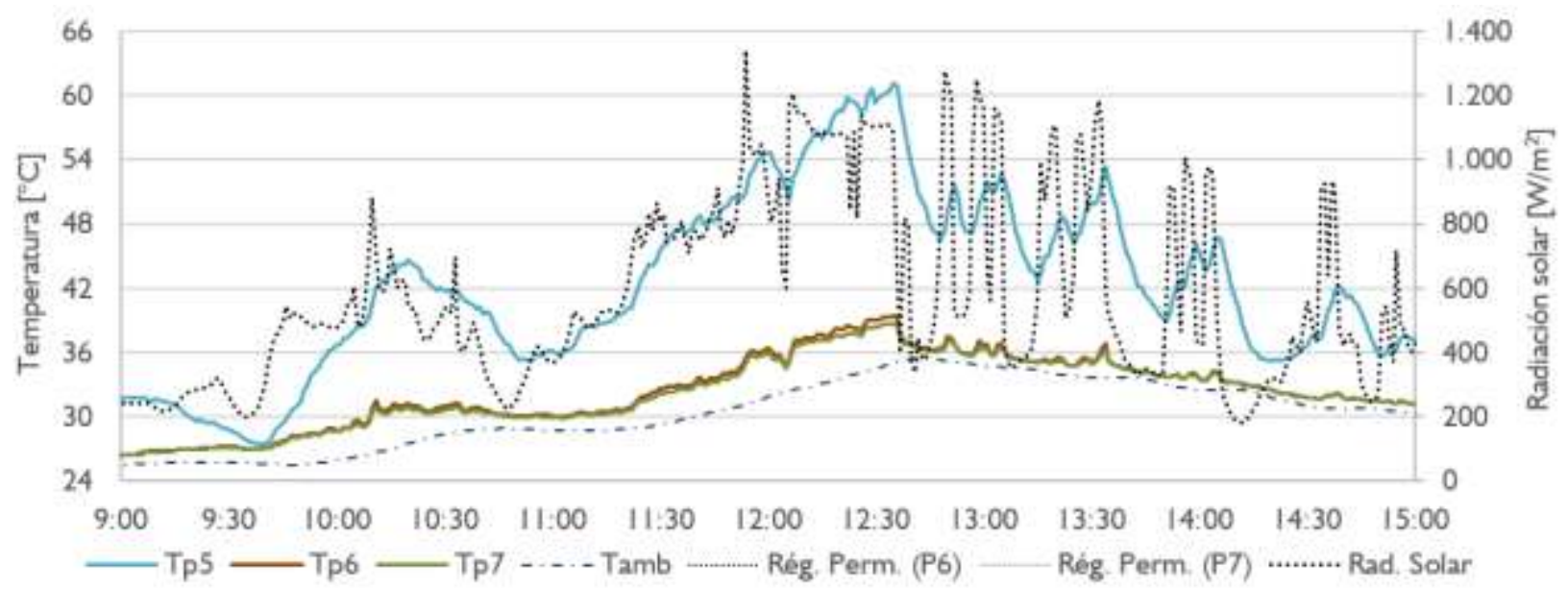

Figura 9. Curvas de las temperaturas de operación de las paneles FV del Caso 7. Fuente. Elaboración propia.

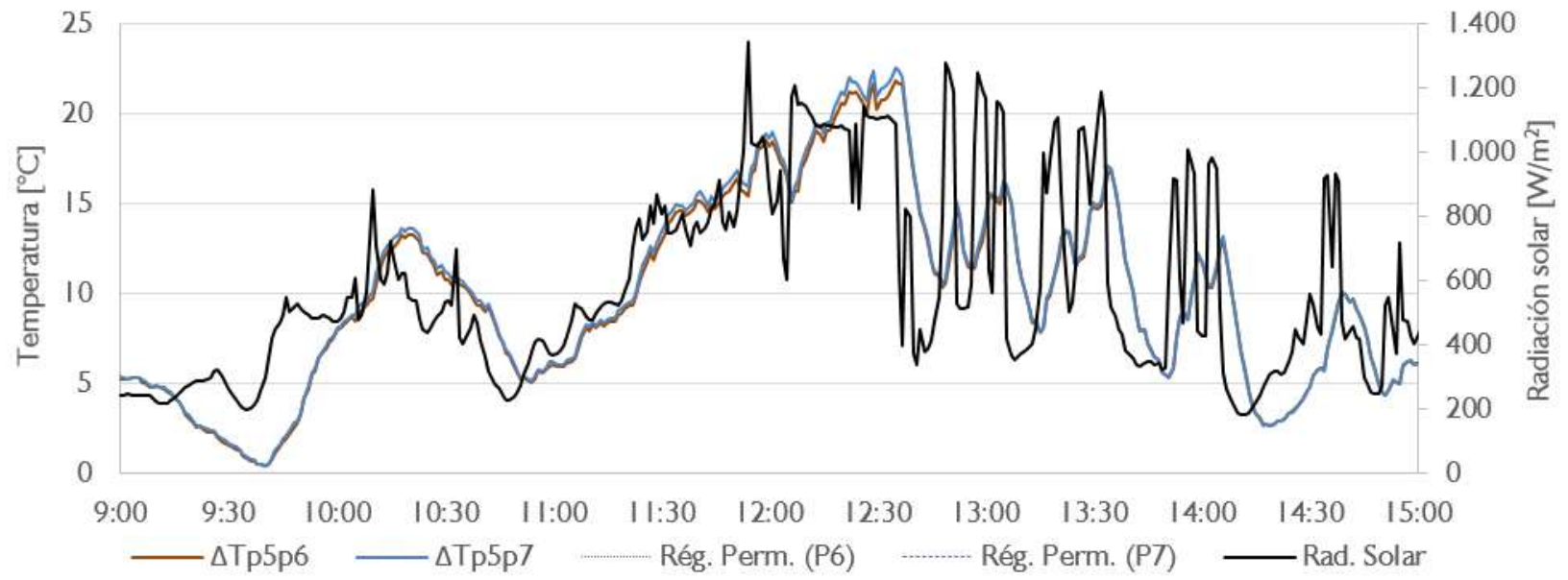

Figura 10. Curvas de decremento de la temperatura de operación de los paneles FV P6 y P7 del Caso 7 con respecto a la temperatura de operación del panel FV P5. Fuente. Elaboración propia.

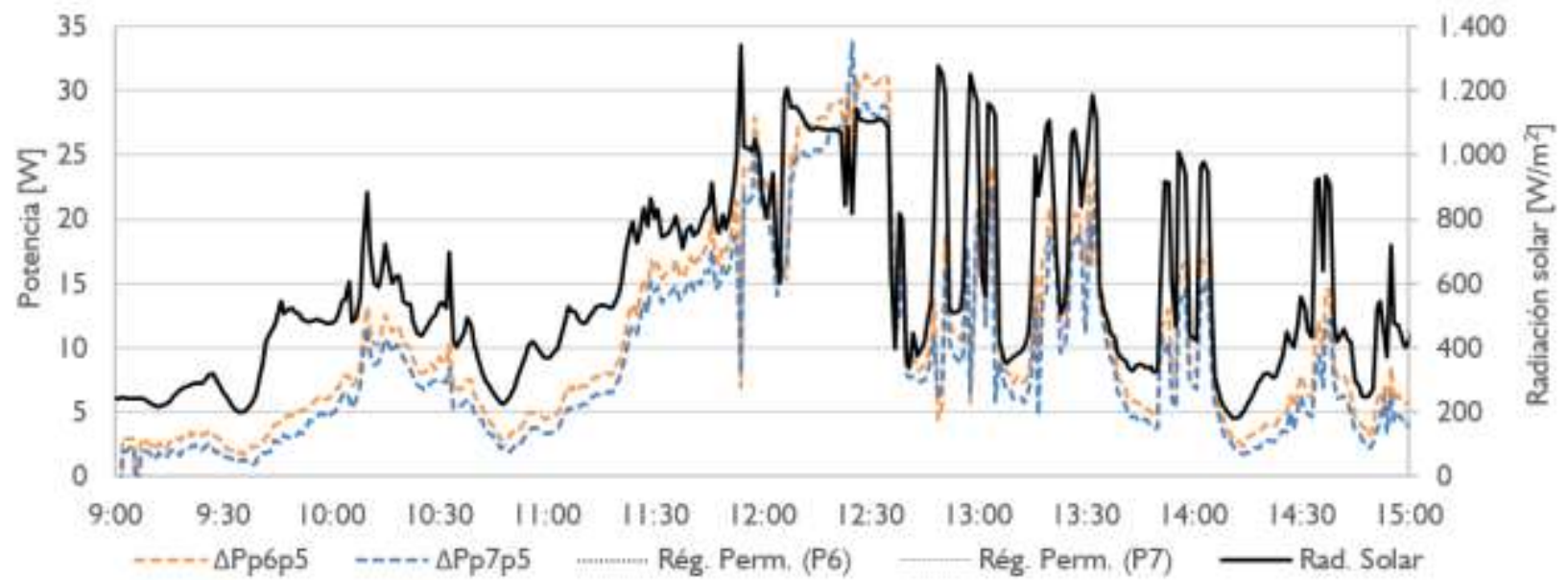

Figura 11. Curvas de incremento de la potencia generada de los paneles FV P6 y P7 del Caso 7 con respecto a la potencia generada por el panel FV P5. Fuente. Elaboración propia. 
Los resultados globales obtenidos de la experimentación se presentan en la Tabla 3, que muestra los valores promedio de disminución de la temperatura de operación y el incremento en la ganancia de energía generada.

Se encontró que el régimen de irrigación de $1 \mathrm{~min}$. puede producir un incremento de entre $0.9 \%$ y $1.4 \%$ en la energía generada diaria para caudales de $3.8 \mathrm{l} / \mathrm{min}$ a 9.5 $1 / \mathrm{min}$, con una disminución de la temperatura de operación entre $2.6^{\circ} \mathrm{C}$ y $4.4^{\circ} \mathrm{C}$.

Para el caso de 15 minutos de irrigación, se observa que la disminución de la temperatura varía entre $6^{\circ} \mathrm{C}$ y $10.5^{\circ} \mathrm{C}$, produciendo un incremento de energía generada entre $4 \%$ y $6 \%$, para caudales entre $3.81 / \mathrm{min}$ y $9.5 \mathrm{l} / \mathrm{min}$. $\mathrm{El}$ incremento para el ciclo de operación permanente es de aproximadamente $9 \%$, para los caudales $4.51 / \mathrm{min}$ y $9.5 \mathrm{l} / \mathrm{min}$.

En general, se considera despreciable el efecto térmico producido por un caudal de $1.8 \mathrm{l} / \mathrm{min}$.

Si se considera el consumo de una bomba sumergible de $8 \mathrm{~W}$, el beneficio energético neto es de $3 \%$ para los ciclos de irrigación de 15 min y permanente, y de sólo $1 \%$ para el caso de 1 min de irrigación.

Tabla 3. Resultados de los experimentos realizados.

\begin{tabular}{|c|c|c|c|c|c|c|c|c|c|c|}
\hline \multirow{2}{*}{$\mathrm{N}^{\circ}$} & \multirow{2}{*}{$\begin{array}{l}\text { Panel } \\
\text { FV }\end{array}$} & \multirow{2}{*}{ Técnica } & \multirow{2}{*}{$\begin{array}{l}\text { Caudal } \\
{[1 / \mathrm{min}]}\end{array}$} & \multirow{2}{*}{$\begin{array}{c}\text { Tiempo } \\
\text { [min] }\end{array}$} & \multicolumn{4}{|c|}{ Temperatura $\left[{ }^{\circ} \mathrm{C}\right]$} & \multicolumn{2}{|c|}{ Ganancia energética } \\
\hline & & & & & Max & Min. & Prom. & Disminución & [Wh/día] & {$[\%]$} \\
\hline \multirow{3}{*}{1} & P5 & Sin riego & - & - & 62,5 & 27,2 & 38,9 & - & - & - \\
\hline & P6 & Película & 9,5 & 1 & 55,3 & 25,3 & 34,5 & 4,4 & 14,3 & 1,4 \\
\hline & P7 & Película & 9,5 & 15 & 51,2 & 25,5 & 31,5 & 7,5 & 40,6 & 4,1 \\
\hline \multirow{3}{*}{2} & P5 & Sin riego & - & - & 58,1 & 28,4 & 44,5 & - & - & - \\
\hline & P6 & Película & 9,5 & 15 & 50,2 & 26,0 & 33,9 & 10,5 & 63,1 & 6,3 \\
\hline & P7 & Chorro $10[\mathrm{~cm}]$ & 3,8 & 15 & 50,9 & 25,4 & 34,9 & 9,6 & 63,9 & 6,4 \\
\hline \multirow{3}{*}{3} & $\mathrm{P} 5$ & Sin riego & - & - & 55,3 & 25,8 & 40,0 & - & - & - \\
\hline & P6 & Chorro $10 \mathrm{~cm}$ & 3,8 & 15 & 49,0 & 23,5 & 31,8 & 7,8 & 41,8 & 4,2 \\
\hline & $\mathrm{P} 7$ & Chorro $20 \mathrm{~cm}$ & 1,8 & 15 & 49,5 & 25,2 & 33,5 & 6,0 & 19,2 & 1,9 \\
\hline \multirow{3}{*}{4} & P5 & Sin riego & - & - & 59,6 & 26,5 & 39,9 & - & - & - \\
\hline & P6 & Chorro $10 \mathrm{~cm}]$ & 3,8 & 1 & 54,6 & 25,6 & 36,0 & 3,9 & 9,2 & 1,1 \\
\hline & P7 & Chorro $20[\mathrm{~cm}]$ & 1,8 & 1 & 54,2 & 26,1 & 37,4 & 2,6 & - & - \\
\hline \multirow{3}{*}{5} & P5 & Sin riego & - & - & 54,9 & 27,4 & 40,8 & - & - & - \\
\hline & P6 & Película & 4,5 & 1 & 54,7 & 26,0 & 36,7 & 4,1 & 12,7 & 1,2 \\
\hline & $\mathrm{P} 7$ & Película & 4,5 & 15 & 49,5 & 25,2 & 32,4 & 8,4 & 43,9 & 4,4 \\
\hline \multirow{3}{*}{6} & P5 & Sin riego & - & - & 60,2 & 29,0 & 45,1 & - & - & - \\
\hline & P6 & Película & 9,5 & 1 & 57,2 & 28,3 & 41,5 & 3,7 & 9,2 & 0,9 \\
\hline & P7 & Chorro $10[\mathrm{~cm}]$ & 3,8 & 1 & 58,4 & 28,9 & 42,4 & 2,7 & 9,4 & 0,9 \\
\hline \multirow{3}{*}{7} & P5 & Sin riego & - & - & 63,3 & 27,2 & 42,2 & - & - & - \\
\hline & P6 & Película & 9,5 & Permanente & 38,7 & 26,3 & 32,3 & 9,9 & 88,9 & 8,9 \\
\hline & P7 & Película & 4,5 & Permanente & 39,3 & 26,7 & 32,5 & 9,7 & 89,9 & 9,0 \\
\hline
\end{tabular}

Fuente. Elaboración propia.

\section{CONCLUSIONES}

Se considera que la irrigación es una estrategia prometedora para mitigar el calentamiento de un panel FV en un entorno tropical cálido. Se encontró que la irrigación de un panel $\mathrm{FV}$, según el experimento ejecutado, puede producir incrementos de hasta $10 \%$ en la generación diaria de energía. Al considerar el consumo energético debido a la bomba de recirculación de agua, el beneficio energético oscila entre $1 \%$ y $3 \%$. Tales valores corresponden a regímenes de operación estáticos durante el día y no en función de la temperatura de operación del panel FV o del nivel de radiación solar incidente.

Se evidenció de forma experimental que a mayor nivel de irradiación solar, mayores son la temperatura de operación de los paneles FV, condición en la cual la mitigación térmica por irrigación es más efectiva. Por el contrario, el efecto de la irrigación es reducido para valores de irradiación solar inferiores a $400 \mathrm{~W} / \mathrm{m}^{2}$ y casi nulo para valores menores a $200 \mathrm{~W} / \mathrm{m}^{2}$. Por tal razón, se considera que la irrigación sólo es potencialmente viable entre 9 a.m. y 4 p.m. 
Este estudio permitió evidenciar la alta incidencia que tiene el régimen de irrigación en el comportamietno térmico de un panel FV y en el beneficio neto energético a ser obtenido.

Se encontró que un caudal de $4.5 \mathrm{l} / \mathrm{min}$ produce el mismo efecto térmico que un caudal de $9.51 / \mathrm{min}$, lo que permiten inferir sobre la existencia de un caudal económico no superior a $4.5 \mathrm{l} / \mathrm{min}$. La determinación de dicho caudal permitirá optimizar el dimensionamiento del sistema de irrigación.

Como trabajo futuro se establece la necesidad de determinar las condiciones necesarias para llevar a cabo la irrigación de un panel FV en función de la obtención de un beneficio energético neto.

\section{AGRADECIMIENTOS}

Los autores agradecen al Departamento Administrativo de Ciencia Tecnología e Innovación - COLCIENCIAS, por el apoyo financiero brindado al proyecto 110274558716 (Contrato No. FP44842-040-2017) titulado "Viabilidad técnica de la implementación de sistemas fotovoltaicos (FV) integrados con vegetación como estrategia de generación distribuida y horticultura en entornos urbanos de clima cálido trópical", el cual se desarrolló según los lineamientos establecidos en la Convocatoria 745 de 2016.

\section{REFERENCIAS}

[1] G. Osma and G. Ordóñez, "Desarrollo sostenible en edificaciones" Rev. UIS Ing., vol. 9, no. 1, pp. 103-121, Jun, 2010.

[2] H. a Hussien, A. H. Numan, and A. R. Abdulmunem, "Improving of the photovoltaic / thermal system performance using water cooling technique," IOP Conf. Ser. Mater. Sci. Eng., vol. 78, p. 12020, 2015.

[3] A. Molki, "Temperature effect on photovoltaic cells," Phys. Educ., vol. 46, no. 5, pp. 523-525, 2011.

[4] J. M. Olchowik et al., "Influence of temperature on the efficiency of monocrystalline silicon solar cells in the South-eastern Poland conditions," Mater. Sci., vol. 24, no. 4, p. 6, 2006.

[5] F. Ju and X. Fu, "Research on impact of dust on solar photovoltaic(PV) performance," 2011 Int. Conf. Electr. Control Eng. ICECE 2011 - Proc., pp. 36013606, 2011.

[6] S. Krauter, "Increased electrical yield via water flow over the front of photovoltaic panels," Sol. Energy Mater. Sol. Cells, vol. 82, no. 1-2, pp. 131-137, 2004.
[7] C. Lamnatou and D. Chemisana, "A critical analysis of factors affecting photovoltaic-green roof performance," Renew. Sustain. Energy Rev., vol. 43, pp. 264-280, 2015.

[8] D. Chemisana and C. Lamnatou, "Photovoltaicgreen roofs: An experimental evaluation of system performance," Appl. Energy, vol. 119, pp. 246-256, Apr. 2014.

[9] H. G. Teo, P. S. Lee, and M. N. a Hawlader, "An active cooling system for photovoltaic modules," Appl. Energy, vol. 90, no. 1, pp. 309-315, 2012.

[10] A.-M. Croitoru and A. Badea, "Air cooling of photovoltaic panels from passive house located inside the University Politehnica of Bucharest," UPB Sci. Bull. Ser. C Electr. Eng., vol. 75, no. 3, pp. 277-290, 2013.

[11] G. George, X. Kanavas, and D. Zissopoulos, "ADAM, intelligent integrated self-enhanced photovoltaic panel with Rainwater harvesting for Irrigation, unit cooling and cleaning," Proc. 24th Int. Conf. Eur. Assoc. Educ. Electr. Inf. Eng. EAEEIE 2013, pp. 174-177, 2013.

[12] H. M. Bahaidarah, S. Rehman, P. Gandhidasan, and B. Tanweer, "Experimental Evaluation of the Performance of a Photovoltaic Panel with Water Cooling," in Photovoltaic Specialists Conference (PVSC), 2013 IEEE 39th. Tampa, Florida, USA, 2013, pp. 2987-2991.

[13] S. Odeh and M. Behnia, "Improving Photovoltaic Module Efficiency Using Water Cooling," Heat Transf. Eng., vol. 30, no. 6, pp. 499-505, 2009.

[14] E. L. Meyer and M. Busiso, "Comparative study of a directly cooled PV water heating system to a naturally cooled module in South Africa," Conf. Rec. IEEE Photovolt. Spec. Conf., pp. 1296-1299, 2012.

[15] A. Kordzadeh, "The effects of nominal power of array and system head on the operation of photovoltaic water pumping set with array surface covered by a film of water," Renew. Energy, vol. 35, no. 5, pp. 1098-1102, 2010.

[16] H. M. S. Bahaidarah, A. A. B. Baloch, and P. Gandhidasan, "Modeling and Comparative Analysis of Jet Impingement Cooling and Conventional Channel Cooling for Photovoltaic Strings," IEEE 40th Photovolt. Spec. Conf., pp. 748-753, 2014.

[17] T. Reindl et al., "Investigation of the Performance of Commercial Photovoltaic Modules under 
Tropical Conditions," Jpn. J. Appl. Phys., vol. 51, p. 4, 2012.

[18] M. Habiballahi, M. Ameri, and S. H. Mansouri, "Efficiency Improvement of Photovoltaic Water Pumping Systems by Means of Water Flow Beneath Photovoltaic Cells Surface," J. Sol. Energy Eng., vol. 137, no. 4, p. 44501, 2015.

[19] M. K. Smith et al., "Water cooling method to improve the performance of field-mounted, insulated, and concentrating photovoltaic modules," J. Sol. Energy Eng. Trans. ASME, vol. 136, no. August, pp. 1-4, 2014.

[20] P. P. Vergara-Barrios, J. M. Rey-López, G. A. Osma-Pinto, and G. Ordóñez-Plata, "Evaluación del potencial solar y eólico del campus central de la Universidad Industrial de Santander y la ciudad de Bucaramanga, Colombia," UIS Ingeniería, vol. 13, no. 2, Jul - Dic, pp. 49-57, 2014. 